\title{
Experimental and numerical studies of hydrodynamics of gas flow through orthogonal networks
}

\section{Grzegorz Walowski ${ }^{a^{*}}$}

${ }^{a}$ Institute of Technology and Life Sciences, Falenty, Al. Hrabska 3, 05-090 Raszyn, Poland

Branch Poznan, Department of Renewable Energy Resources, 67 Biskupińska Street , 60-463

Poznań, Poland,

e-mail address: walowski.g@gmail.com or g.walowski@itp.edu.pl

* Corresponding author

\begin{abstract}
Simulation programs contain Computational Fluid Dynamics - CFD codes and are a useful tool used for gas flow through porous materials. Conducting numerical simulations allows for detailed analysis of hydrodynamic phenomena. The results of numerical modeling should always be verifiable based on experimental data. Only their compliance with the results of experimental tests is a determinant of the correctness of the applied method. As part of the work, experimental studies of hydrodynamics of gas flow through an isotropic porous material were carried out and numerical simulation for material of the same shape was used. In the CFD modeling Kolmogorov's hypothesis for the transport of kinetic energy of turbulence $\mathrm{k}$ and transport of dissipation rate of kinetic energy of turbulence $\varepsilon$ was used.
\end{abstract}

Keywords: isotropic material, CFD, hydrodynamics, gas flow 


\section{List of major signs}

a edge of the cube $\quad \mathrm{m}$

$b \quad$ vector of mass forces (acceleration of gravity) $\mathrm{m} / \mathrm{s}^{2}$

c constant

diameter

$\mathrm{m}$

e unit base vector

$k \quad$ kinetic energy of turbulence

$\mathrm{m} / \mathrm{s}^{2}$

$l \quad$ scale of vortex

$p$

pressure

$\mathrm{Pa}$

$t$

time

S

u velocity

$\mathrm{m} / \mathrm{s}$

$x$

Cartesian coordinate

D

outer diameter of the cell

$\mathrm{m}$

$L$

flow path

$\mathrm{m}$

$P$

pressure

$\mathrm{Pa}$

$Q$

stream

$\mathrm{m}^{3} / \mathrm{s}$

Re Reynolds number

$T \quad$ scale of tunnel

V

volume

$\mathrm{m}^{3}$

$\Delta P$

pressure drop, resistance flow

$\mathrm{Pa}$

$\delta$

Kronecker symbol

$\varepsilon$

dissipation of kinetic energy of turbulence

$\mathrm{m}^{2} / \mathrm{s}^{-3}$

$\eta$

fluid viscosity

$\mathrm{Pa} \cdot \mathrm{s}$

$\lambda$

the Kolmogorov scale

$\rho$

fluid density

$\mathrm{kg} / \mathrm{m}^{3}$ 
$\tau$

$\psi \quad$ indicator porosity

$\omega$

$\vartheta$

$\Psi$

stress

vortex

porosity
$\mathrm{Pa}$

density of a solid body

\section{Lower indices refer to}

$\begin{array}{ll}1,2,3 & \text { Cartesian components } \\ b & \text { absolute } \\ e & \text { equivalent } \\ g & \text { gas } \\ k & \text { ball } \\ o & \text { reference } \\ p o z & \text { apparent } \\ p r & \text { sample } \\ \text { st } & \text { skeleton } \\ \text { swo } & \text { free } \\ z m & \text { measured }\end{array}$

\section{Introduction}

As a principle, theoretical considerations with regard to the gas flow through porous media are based on the models of flow through axial microchannels, capillaries by application of the laws pertaining to flows, including Poiseuille'a law - as well as the indications of the pressure drop applying simplifying assumptions. However, in each case, the models are based on the quantities describing the physical characteristics of the porous medium, such as: shape of pores, their size and arrangement and bed permeability. 
The motion of a viscous fluid is described by the Navier-Stokes - N-S equations $(1 \div 3)$ which along auxiliary equations $(4 \div 5)$ and the continuity equation (6) form the comprehensive system of relations applied to derive the pressures and velocity profile of the fluid flow [1]:

$\frac{\partial(\rho u)}{\partial \tau}+(u \cdot \nabla)(\rho u)=-\nabla p+\eta \nabla^{2} u+\rho b$

where the velocity vector and specific base vectors are functional relations

$$
\begin{aligned}
& u=u_{1} e_{1}+u_{2} e_{2}+u_{3} e_{3} \\
& p=p\left(x_{1} x_{2} x_{3}\right) .
\end{aligned}
$$

whereas the vector Hamilton (4) and Laplace (5) operators take the form

$$
\begin{aligned}
& \nabla=\frac{\partial}{\partial x_{1}} e_{1}+\frac{\partial}{\partial x_{2}} e_{2}+\frac{\partial}{\partial x_{3}} e_{3} \\
& \nabla^{2}=\frac{\partial^{2}}{\partial x_{1}^{2}} e_{1}+\frac{\partial^{2}}{\partial x_{2}^{2}} e_{2}+\frac{\partial^{2}}{\partial x_{3}^{2}} e_{3}
\end{aligned}
$$

The continuity equation (6):

$$
\frac{\partial \rho}{\partial t}+\nabla \cdot(\rho u)=0
$$

By adopting the Reynolds hypothesis which offers the notation of the components of the velocity vector (7) and pressure field (8) as the scalar totals of the averaged values, we obtain:

$$
\begin{aligned}
& u_{i}=\bar{u}_{i}+u_{i}^{\prime} \\
& p_{i}=\bar{p}_{i}+p_{i}^{\prime}
\end{aligned}
$$

Depending on the particular needs and the type of the investigated problem, e.g. incompressible and compressible fluid, there are different ways in which the averaging of this system of equations is performed. Although the numerical solutions need to apply a very thick mesh discretization with regard to the examined flow area, the up-to-date computer 
techniques provide solutions to the problems which are a few orders of magnitude smaller from the requirements of the current engineering practice. As a consequence, the applied calculation models require considerably smaller network discretization.

Most commonly this objective applies equations averaged over time or space, suitably to the method based on the Reynolds hypothesis, as for instance based on the $k-\varepsilon$, model or a small scale approximation methods suited to model Large Eddy Simulation - LES. The averaging of the velocity profile in time leads to the derivation of Reynolds equations expressing the law of the conservation of momentum in averaged turbulent flow [2].

The reason for the application of the models of the turbulent motion - which area usually more complex than the clear $N-S$ model is associated with the scale problem which needs to be solved for the case of the gas flow, as it is schematically described in Fig. 1.

Fig. 1. Scale of the problem for numerical models of turbulent flow based on [1]: $\lambda$-Kolmogorov scale, $l$ - vorticity scale, $T$ - channel scale.

The scope of the calculations needed to apply a model is relative to this scale. On the Kolmogorov scale $\lambda$ in the $N-S$ equation $(1 \div 3)$, the dominant role is attributed to the term derived from viscosity. The gas motion occurs in the laminar conditions and it is characterized by a considerable level of energy dissipation, which is converted into heat. On the vorticity scale $l$, the gas motion occurs in the conditions where the term representing the viscosity in the $N-S$ equation $(1 \div 3)$ is close to being disregarded. This, in turn, leads to motion of gas driven by inertia, as the energy of the motion is extracted from the spatially extensive areas: it passes through a cascade of vortices without its dissipation in the areas with the smaller capacity. For larger scales in space - on the channel scale - there are coherent structures in the form of vortices, which partly synchronize their pace of motion. On this scale, forces which induce motion occur and this motion is driven by means of large-scale structures. 
Under the assumption that a fluid has homogenous and incompressible properties $\rho=$ const., $\eta=$ const., averaging over time gives (9):

$$
\bar{u}(x, \tau)=\frac{1}{\Delta \tau} \int_{t}^{t+\Delta t} u(x, \tau) d \tau
$$

by application of the continuity equations $(6)$, this leads to the Reynolds hypothesis $(7 \div 8)$ this leads to an equation which relates the mean values of the components of the velocity vector (10) in the form:

$$
\nabla \cdot \bar{u}=\sum_{i=1}^{3} \frac{\partial \bar{u}_{i}}{\partial x_{i}}=\frac{\partial \bar{u}_{i}}{\partial x_{i}}=\bar{u}_{i, j}=0
$$

The substitution into $(1 \div 3) N-S$ gives $(11)$ :

$$
\frac{\partial \bar{u}_{i}}{\partial t}+\bar{u}_{j, i} \bar{u}_{i}=-\frac{1}{\rho} \bar{p}_{j}+\left(\bar{\tau}_{i j}+\tau_{i j}^{*}\right)_{i}
$$

where

$$
\begin{aligned}
& \bar{\tau}_{i, j}=\eta\left(\bar{u}_{i, j}+\bar{u}_{j, i}\right) \\
& \tau_{i j}^{*}=-\rho \overline{u_{i}^{\prime} u_{j}^{\prime}}
\end{aligned}
$$

denote the strains occurring in the fluid.

The Reynolds stress tensor defined by equation (12) results in additional difficulties in the solution of the models based on such averaging Reynolds Averaged Naver-Stokes equations - RANS, as it adds new unknown values in the system of equations $(1 \div 6)$; as a result, it is no longer in the closed form. This also requires the application of further equations to express the components of the tensor $\tau_{i j}^{*}$ described by the equation in (13).

A compromise in these circumstances can be sought in the application of the $k$ - $\varepsilon$ model. It was first proposed by Chou [3], and further modified on multiple occasions [4]; hence, it is 
currently one of the most commonly used turbulence model with regard to incompressible fluids with small velocities.

The Reynolds stress tensor for turbulent flow is defined by an additional equation in this model (14):

$$
\tau_{i j}^{*}=\eta_{T}\left(\bar{u}_{i, j}+\bar{u}_{j, i}\right)-\frac{2}{3} \bar{\rho} k \delta_{i j}
$$

for

$$
\eta_{T}=\rho c_{\mu} k^{2} / \mathcal{E}
$$

Despite the fact that the $k$ - $\varepsilon$ model is strictly dedicated to incompressible flow, this model is successfully applied in the area of gas flow, in particular in the condition when solutions regarding the impact of the local disturbance on the hydrodynamic parameter of the fluid flow are investigated. The concept of this model uses the closure of the equations $(1 \div 3)$ $N-S$ with two additional differential equations for the kinetic energy transfer of turbulence k and the transport of turbulence kinetic energy dissipation rate $\varepsilon$, which can be estimated using the Kolmogorov hypothesis [5]. This hypothesis indicates the relation between the macroscopic flow structures expressed by a linear scale of the flow accounting for the dissipation, which characterizes even the slightest vortex scales.

We can note here that another model in the RANS group, namely $k$ - $\omega$ model, also forms a model of the transport of the kinetic energy of turbulence $k$, which in this case is linked to the vorticity $\omega$. In this case, the system consists of two equations. However, its application with regard to various types of flows does not offer satisfactory solutions due to the adoption of an assumption regarding the scalar nature of the vortex viscosity [6]. 


\section{Materials and metod}

This research material Fig. 2a directly comes from the polymerization of spherical grained powders Fig. 2b (manufacturer: Przedsiębiorstwo Wdrażania Innowacji "INWET" S.A.) and is used as the so-called gas distributor designed for aerating liquid systems; other names are porous polyethylene or porous polyamide, depending on the type of plastics applied.

Fig. 2. Research material - porous sinter: a) cube $20 \times 20 \times 20 \mathrm{~mm}$, b) porous structure (black: free space; white: frame-based structure).

The apparent density $\vartheta_{p o z}$ of the porous material was determined by measuring the total volume of the $V_{p r}$ sample (by immersing it into water) and its mass, which in this case corresponds to the mass of the solid body $m_{p r}=m_{s t}$. Definition (16):

$\vartheta_{p o z}=\frac{m_{s t}}{V_{p r}}$

The absolute porosity of the porous material $\Psi_{b}$ is calculated on the basis of the quotient of the free volume $V_{\text {swo }}$ and the total volume of the sample $V_{p r}$. Following some relevant transformations this definition may be as follows (17):

$\Psi_{b}=\frac{V_{s w o}}{V_{p r}}=1-\frac{V_{s t}}{V_{p r}}=1-\left(\frac{m_{s t}}{\vartheta_{s t}}\right)\left(\frac{\vartheta_{p o z}}{\vartheta_{s t}}\right)=1-\frac{\vartheta_{p o z}}{\vartheta_{s t}}$

Determined for porosity rate $\psi$ that, on the other hand, characterises a density degree of the porous medium [7]. Definition (18):

$\psi=\frac{\Psi_{b}}{1-\Psi_{b}}$

The parameters determined for the tested materials (samples) are set forth in Table 1 together with sample numbers and average values of those parameters.

\section{Table 1}

Characteristic of research material 
In Table 1 contains a characteristic of sample of porous material, along with the information regarding the multiple of the volume of the cube-shaped sample with the dimensions of $20 \times 20 \times 20 \mathrm{~mm}$.

\subsection{Experimental research}

To obtain the research objective, the detailed experimental tests were conducted to assess the gas permeability of porous material.

The measurement system - Fig. 3 - was applied to test the permeability of tests were conducted on cubic-shaped samples. In this system the gas flow was always directed with respect to the arbitrarily selected solid axis X, Y, Z. In each case, gas flew through the sample in the free-pressure regime, the reference pressure in the pressure reducing valve was $(0.04 \div 0.16) \mathrm{MPa}$ and gas freely outflew. Air was taken from the compressed air system at the reference temperature of $18^{\circ} \mathrm{C}$. The decline in pressure in a specific measurement system was measured with differential pressure manometers installed in the stream measurement system at the gas inlet to the material sample. The gas stream was measured with flota-controlled rotameters scaled with the bellows gas meter before conducting the tests. All the results of the gas stream measurements were referred to normal conditions (293 K, $1013 \mathrm{hPa})$.

Fig. 3. Scheme of measurement system for porous material permeability tests for directed gas flow: 1 - porous material (sample), 2 - differential pressure manometer, 3 - rotameter (3abubble flowmeter), 4 - pressure redactors, 5 - control valve, $\mathrm{P}$ - manometer, $\mathrm{T}$ thermometer.

The measurement system, more strictly the construction of the permeability meter [8] employed in the directed gas flow along the selected sample's axis X, Y, and Z, used for the cubic-shaped samples, is described in Fig. 4. 
Fig. 4. Gas permeability meter: a) view, b) construction scheme:

1- housing, 2- cylindrical body, 3- gas inlet pipe, 4- bottom base, 5- bottom gasket, 6motionless external bottom part, 7- threaded rod, 8- motionless internal bottom part, 9bottom back-up ring, 10- measurement cell, 11- porous material sample, 12- upper back-up sample, 13- movable upper bush, 14- nut, 15- upper base, 16 - upper gasket, 17 - gas outlet pipe.

This permeability meter [8] is designed for testing the permeability of samples in the form of a cubic solid in any direction of its axis, which is possible by applying a special sealing system of the measuring cell that enables measuring the permeability towards any direction $\mathrm{X}$, $\mathrm{Y}, \mathrm{Z}$ of the location of the measurement sample in the measurement system according to the scheme as shown in Fig. 3.

The scheme of this measurement cell together with the marking of the sealing material and the measurement sample is shown in Fig. 5.

Fig. 5. Scheme of measurement cell: 1 - porous material, 2 - sealing material (cubic sample); $\mathrm{D}=49 \mathrm{~mm}, \mathrm{a}=20 \mathrm{~mm}$.

\subsection{Numerical research}

Regardless of the method that is applied, the numerical calculations need to involve the derivation of a geometrical representation of the modeled object. In ANSYS Fluent, this is performed in the DesignModeler module [9].

The numerical network should fitfully reproduce the entire area occupied by gas by ensuring an adequate fineness ratio of the microchannel shape and its volume so as to consider it as a single computing cell. In this, it is necessary to ensure that a compromise is sought between the level of detail and the number of nodes in the numerical network, which affects the calculation time.

The network generation involves the discretization of the model space, i.e. the description how it can be divided into finite elements, which define the so called control 
volume. Each element of the larger whole expressed by the geometry forms a space in which gas flow is balanced in accordance with the equations defining the preservation of mass, momentum and energy. In the literature in the field [10], it is stressed that the numerical network forms one of the most important aspects of the model development, since its density determines the precision of the calculations and time needed to establish a solution. This network should also assume the greatest density in the areas in which the exchange processes should occur, such as the occurrence of turbulent motion and abrupt change in the flow directions.

The network generation applies a dedicated module named Mesh in the ANSYS Fluent program. This software is applied for the data preparation and gaining numerical solutions to complex issues in the area of gas transport as a result of Solver Preference module in Fluent. The accuracy of the output is basically relative to the adequate method selection - in this case it is Insert Method. In this procedure, it is valuable to select the Sweep calculation option, as this affects the structure of the elements forming the grid during its generation with regard to the microchannel.

The standard $k-\varepsilon$ model requires the application of considerably smaller discretization networks, as equations $(1 \div 3) \mathrm{N}-\mathrm{S}$ averaged in time are applied in this case. The averaging $\mathrm{n}$ time of the velocity field leads to the statement of the Reynolds equation, given that (19):

$R e=\frac{u d \rho}{\eta}$

The number of nodal points on an adequate spatial network should in this case be proportional to $\mathrm{Re}^{9 / 4}$, which results from the statistical theory of the turbulent motion $[11,2]$. 


\section{Results and discusion}

\subsection{Experimental assesment}

A reference point to the assessment of hydrodynamics of gas flow through a porous material was a porous polyamide comprising the agglomerate of spherical particles with identical dimensions, about $0.1 \mathrm{~mm}$ diameter. This material has the absolute porosity of $32 \%$ Table 1 and its structure is similar to the rhombus-shaped system.

To obtain the research objective, the detailed experimental tests were conducted to assess the gas permeability of skeletal material porous by using air as a working medium Table 2 .

\section{Table 2}

Test of research material.

The distribution of the experimental points shown in Fig. 6 proves that the permeability characteristics of this material do not practically depend on a direction of the gas flow, which proves the symmetrical structure of its construction and isotropic properties. Interestingly, for the porous polyamide, the permeability characteristics are nonlinear in nature, which in the range of measurements indicates the predominance of turbulent gas flow over its laminar flow.

Fig. 6. Distribution of experimental points characterising asymmetry of air flow through deposit of polyamide cubic-shaped sample with dimensions of $20 \times 20 \times 20 \mathrm{~mm}$.

\subsection{Numerical assesment}

The characteristic type of polyamide material is shown in Fig.7, which in its structure is representative of the microchannels forming the orthogonal network - typical for polyamide sinter.

The network model of the geometry of gas flow forms the structural equivalent to these materials, as it represents the structure and porosity of the materials in question. The porous 
sinter (polyamide) demonstrating the characteristics of isotropy Fig. 6 forms in this respect a practical model of the porous material whose equivalent number for a sample volume with the cubic shape is equal to $n=1$ Table 1 . In addition, a porous medium characterized by a porous structure Fig. 7a is repeatable in terms of its layout of all its components, which leads to the conclusion about repetitive geometry of the calculation network along its entire volume.

Fig. 7. Geometrical model of porous bed with a symmetric cube structure $a=20 \mathrm{~mm}, d_{k}=10 \mu \mathrm{m}, \Psi=32.2 \%$ :

a) elementary bed model structure, b) geometrical structure of symmetric porous spaces.

On the basis of the model of the geometry of porous spaces Fig. 7b, a specific gas flow unit was identified to establish the elementary model of its geometry - Fig. 8a. This was followed by its discretization for various densities of the grid applied in the calculations, which is presented by examples in Fig. 8b. From the assessment of the impact of the number of grid cells on pressure distribution in the orthogonal network it follows that the repeatable results of calculation are obtained for $85 \cdot 10^{3}$ and more number of cells - Fig. 9 .

Fig. 8. Elementary unit of cellular open for flow structure: a) model of geometry, b) calculation network $-85 \cdot 10^{3}$ cells.

Fig. 9. Discretization of model of geometry for cube-shaped network $a=20 \mathrm{~mm}, d_{k}=10 \mu \mathrm{m}, \Psi=32.2 \%$.

The purpose of analyzing the hydrodynamics of the gas flow through microchannels in a porous material needs to include a stage concerned with defining the material properties, setting the conditions of the environment and choosing the boundary conditions. Such a stage forms one of the most important aspects of applying the $C F D$ method, as the range of the initial and boundary conditions considerably affects the quality of the resulting numerical 
solution. Following this stage, it is possible to initiate the calculations and gain calculation images of the pressure and velocity fields.

The analyzed issue involved the definitions of superficial criteria needed to determine the boundary conditions at the inlet: pressure - inlet, velocity - inlet. For this purpose, selected areas were identified along the model object and further referred to as zones. Such zones are considered in the calculation procedure to represent the boundaries, for which selected input quantities, both the measurable as well as observable ones are defined. The boundary condition at the inlet was defined to be the air velocity at the channel inlet with the value of $u_{g}=0.195 \mathrm{~ms}^{-1}$. The boundary condition for the outlet pressure was adopted to be given by the static pressure.

The results of the calculations regarding pressure variations accompanying gas flow through a structural cube-shaped system are presented in Fig. 10a. The same calculation criteria were applied for the description of the velocity field, and the results are presented in Fig. 10b. For a specimen with the multiple volumes, the results are presented in Fig. 11 for analogy purposes. In each of these cases, we obtain a correct set of calculation results, which indicates that the adopted methodology of modeling the geometric structure of porous materials is a correct one.

The summary of the results, yet referring to the comparison of the results of the numerical and experimental data, is presented in Fig. 12.

Fig. 10. Distribution of gas flow in the framework structure for the cross-section of the elementary unit: a) pressure field, b) velocity field.

Fig. 11. Distribution of gas flow in the framework structure:

a) pressure field for the 15-times multiple of the elementary unit, b) direction lines of fluid flow and velocity profiles for 5-times multiple of the elementary unit. 
The results of the numerical studies - which are related to the mean structure of these materials - offer a suitable characteristic of the conditions resulting from the hydrodynamics of the gas flow through a porous bed. The tendency to note variations in the permeability function that was observed - for a constant geometric network represented by the velocity of the gas flow offers a considerable degree of repeatability is does not present distinct results from the ones gained from the experiment.

The lower results gained from the experiment for the case of polyamide Fig.12 are undoubtedly associated with the imperfections of the structure of this material, formed by sintered material with a cube-shaped structure, as it comprises a large number of defects in the capillary flow channel, which result from the characteristics of the sintering process. This could lead to formation of an asymmetric structure, which was not accounted for in the numerical calculations.

Fig. 12. Summary of experimental and numerical results for porous sinter.

The analysis of the results presented in Fig. 12 also leads to the statement that for each type of material, there must be a boundary range of gas velocities for which the pressure drop assumes the lowest value for around $1 \mathrm{~ms}^{-1}$ for polyamide. This distinction is undoubtedly associated with the diverse structure of these materials. The results confirm the correctness of the adopted calculation methodology and provide an opportunity to assess the hydrodynamics of gas flow through the porous structures.

This study can form a considerable contribution to the validation of the results of testing performed in the conditions of actual porous beds. 


\section{Conclusion}

Experimental research has proven that a porous sintered polyamide is an isotropic material. The results of numerical simulations enabled the quantitative analysis of hydrodynamics of gas flow through the structure of orthogonal micrograns. Compatibility of calculations with experimental data is a prerequisite for the application of the $C F D$ method in studies of hydrodynamic phenomena, taking into account Kolmogorov's hypothesis.

\section{Acknowledgments}

The study conducted as part of the project financed by the National Centre for Research and Development conducted in the BIOSTRATEG program, contract No BIOSTRATEG1/269056/5/NCBR/2015 dated 11 August 2015.

\section{References}

[1] Ferziger JH, Peric M. Computational Methods for Fluid Dynamics. Springer, 1999.

[2] Murakami S, Mochida A. Applications of CFD to bluff body aerodynamics. A State of Art in Wind Eng. New Delhi, Wiley Eastern Limited: Ninth International Conference on Wind Engineering. A State of the Art in Wind Eng. 1995; 65-89.

[3] Chou (also Zhou) PY. On velocity correlations and the solutions of the equations of turbulent fluctuation. Quart. Appl. Math. 1945;3:38-54.

[4] Launder BE, Reece GJ, Rodi W. Progress in development of a Reynolds-stress turbulent closure. J. Fluid Mech, 1975;68:537-566.

[5] Elsner JW: Turbulence flows. Warszawa, PWN, 1987.

[6] Bogusławski A, Drobniak S, Tyliszczak A. Turbulence - from randomness to determinism. Engineering modeling. Gliwice 2008; 36:41-48. 
[7] Cała M. Soil mechanics. Department of Geomechanics, Civil Engineering and Geotechnics. AGH University of Science and Technology, Krakow. Retrieved December, 1, 2012 from http://home.agh.edu.pl/ cala/prezentacje/1wyklad_ZG.pdf.

[8] Filipczak G, Wałowski G, Krause E. System for determination the coefficient of gas permeability through porous materials with anisotropic structure, preferably through chars. Patent application P.409191, Patent Office of the Republic of Poland, Warsaw 2014.

[9] Ansys Inc. 2010 - Academic Research, Relase 13.0,. Help System, CFX- Solver Theory Guide 2010

[10] Siuda T: Possibilities of using the FLUENT program in the works carried out at the Oil and Gas Institute. Kraków, Nafta-Gaz, January, year LXVII, 2011; 1:53-63.

[11] Chung TJ: Computational Fluids Dynamics. Cembridge University Press 2002. 




Fig. 1. Scale of the problem for numerical models of turbulent flow based on [1]: $\lambda$-Kolmogorov scale, $l$ - vorticity scale, $T$ - channel scale. 
a)

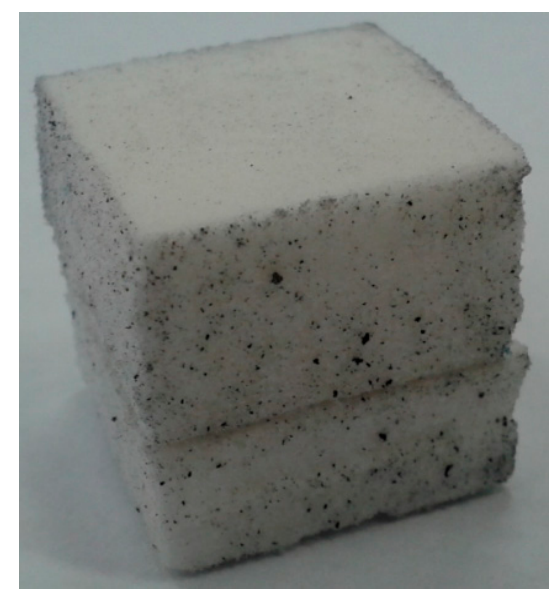

b)

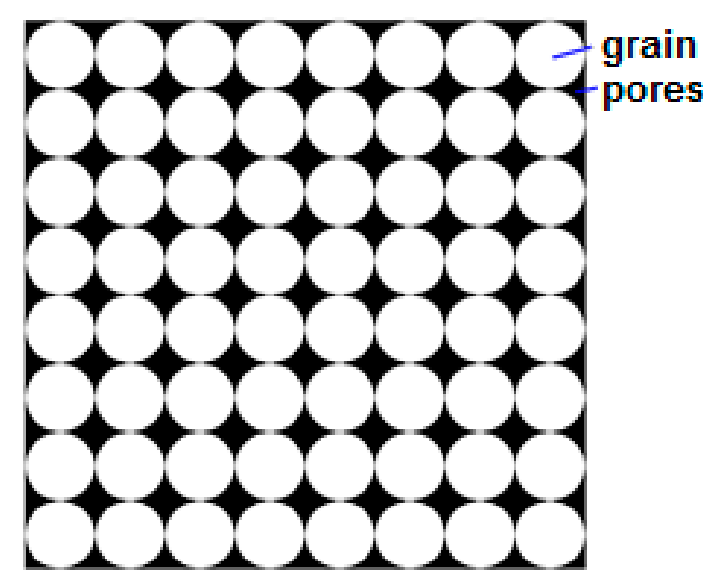

Fig. 2. Research material - porous sinter: a) cube $20 \times 20 \times 20 \mathrm{~mm}$, b) porous structure (black: free space; white: frame-based structure). 


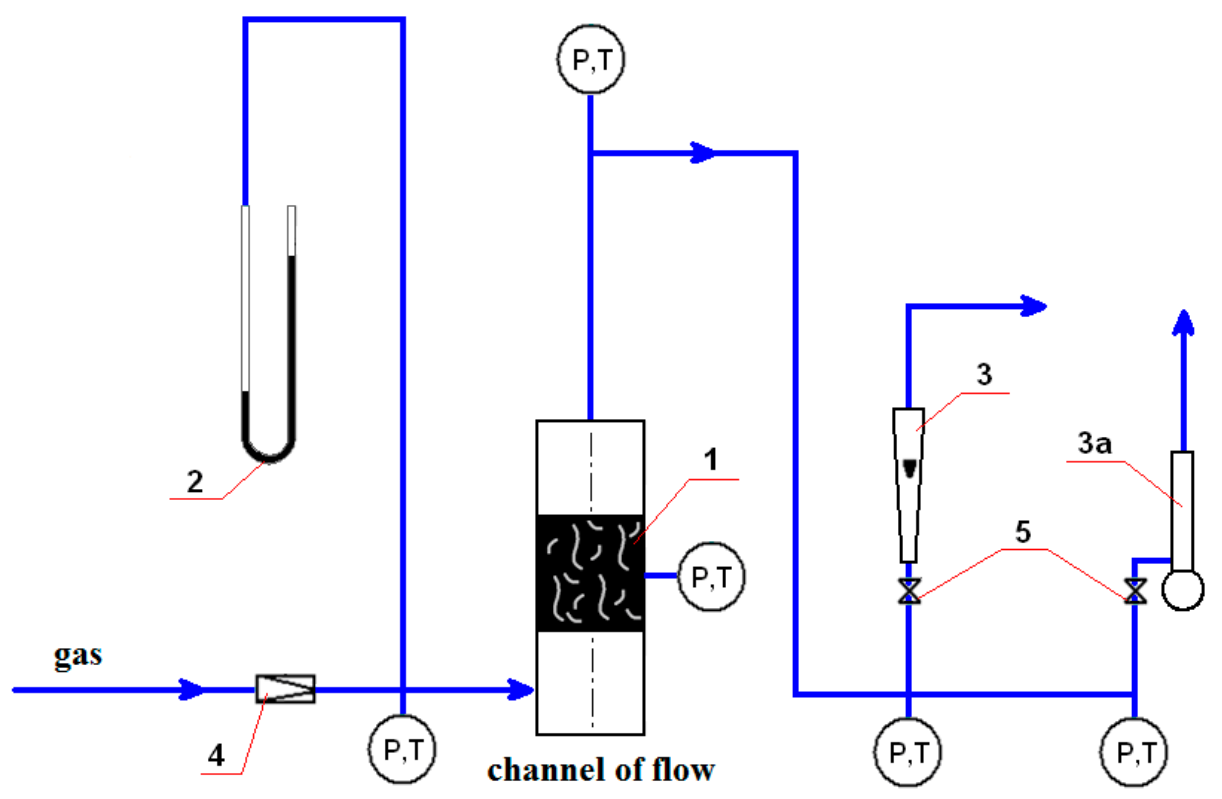

Fig. 3. Scheme of measurement system for porous material permeability tests for directed gas flow: 1 - porous material (sample), 2 - differential pressure manometer, 3 - rotameter (3abubble flowmeter), 4 - pressure redactors, 5 - control valve, $\mathrm{P}$ - manometer, $\mathrm{T}$ thermometer. 
a)

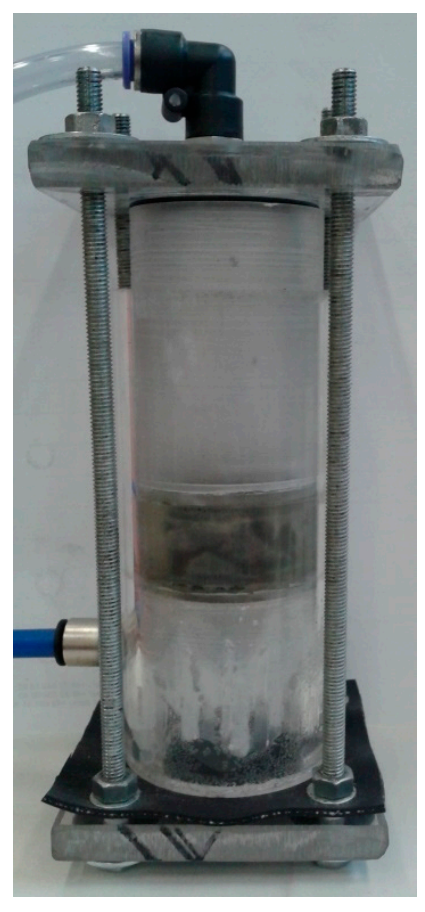

b)

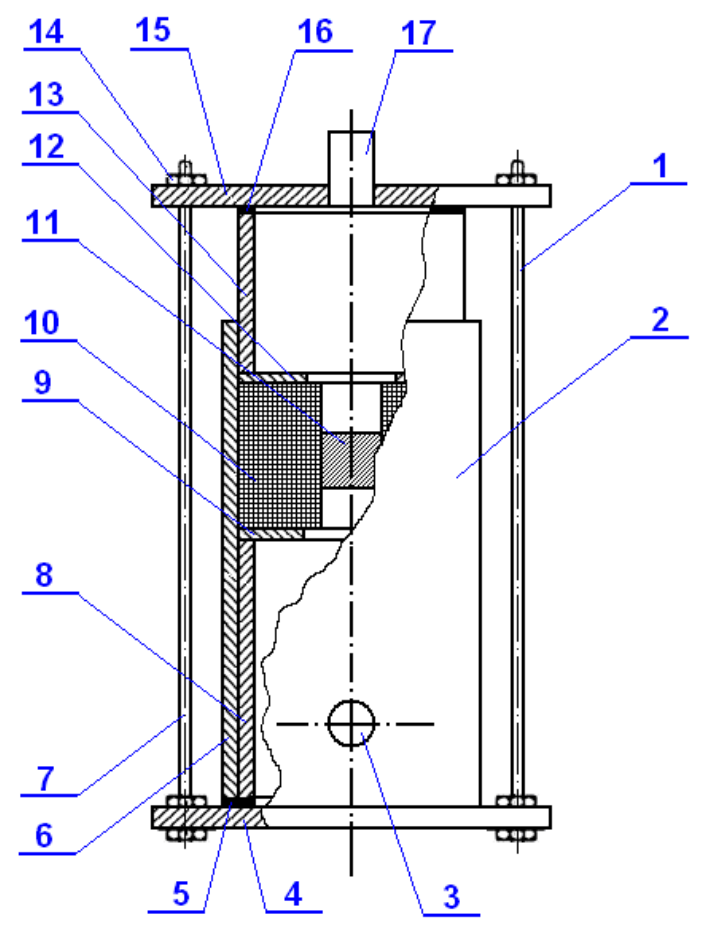

Fig. 4. Gas permeability meter: a) view, b) construction scheme:

1- housing, 2- cylindrical body, 3- gas inlet pipe, 4- bottom base, 5- bottom gasket, 6motionless external bottom part, 7- threaded rod, 8- motionless internal bottom part, 9bottom back-up ring, 10- measurement cell, 11- porous material sample, 12- upper back-up sample, 13- movable upper bush, 14- nut, 15- upper base, 16 - upper gasket, 17 - gas outlet pipe. 


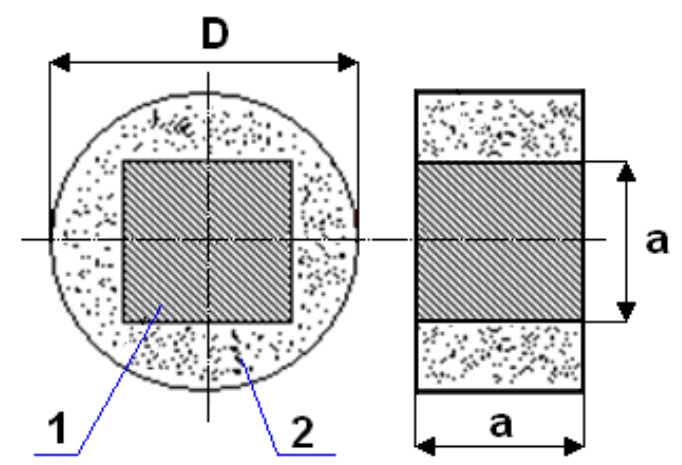

Fig. 5. Scheme of measurement cell: 1 - porous material, 2 - sealing material (cubic sample); $\mathrm{D}=49 \mathrm{~mm}, \mathrm{a}=20 \mathrm{~mm}$. 


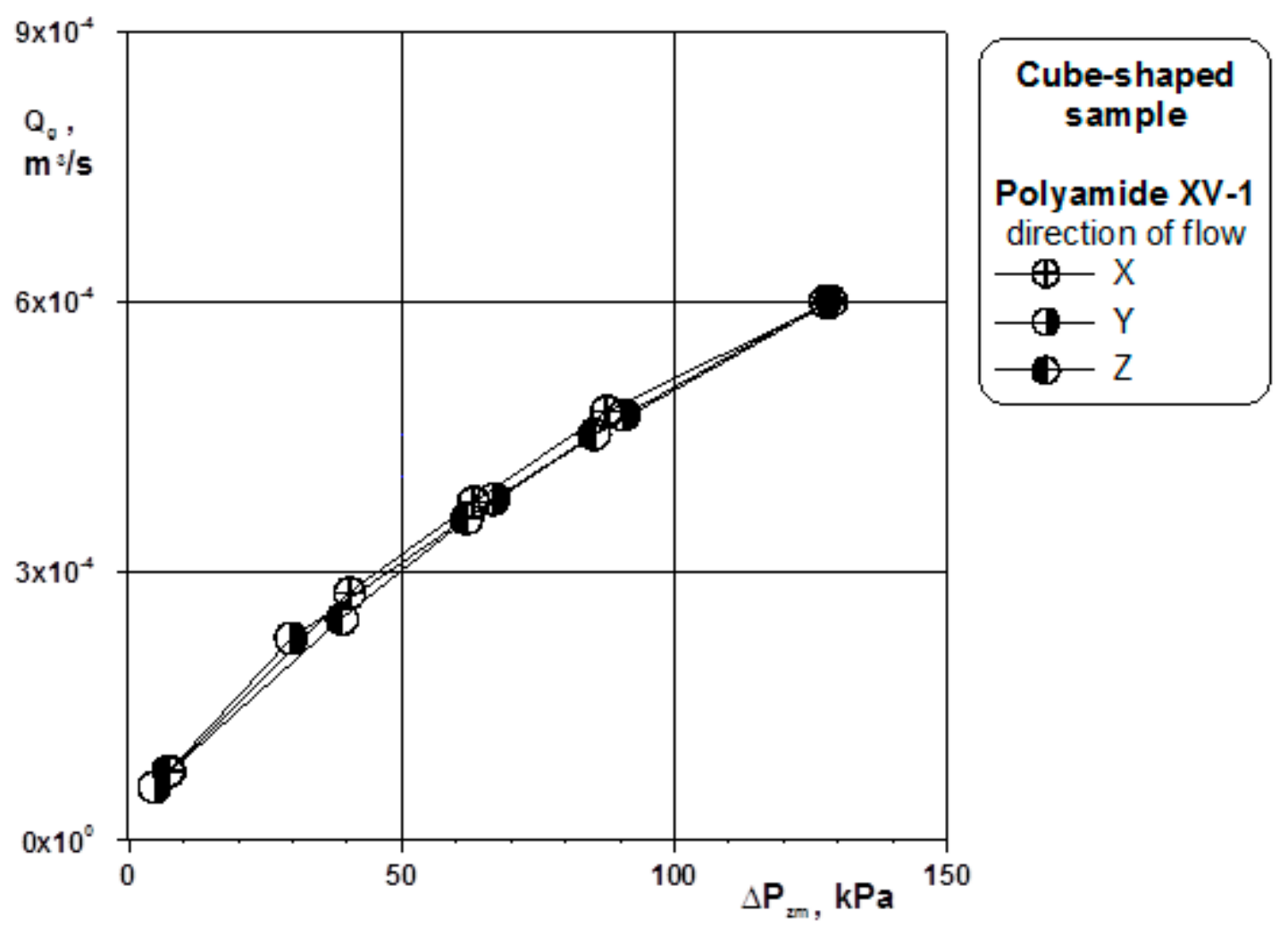

Fig. 6. Distribution of experimental points characterising asymmetry of air flow through deposit of polyamide cubic-shaped sample with dimensions of $20 \times 20 \times 20 \mathrm{~mm}$. 
a)

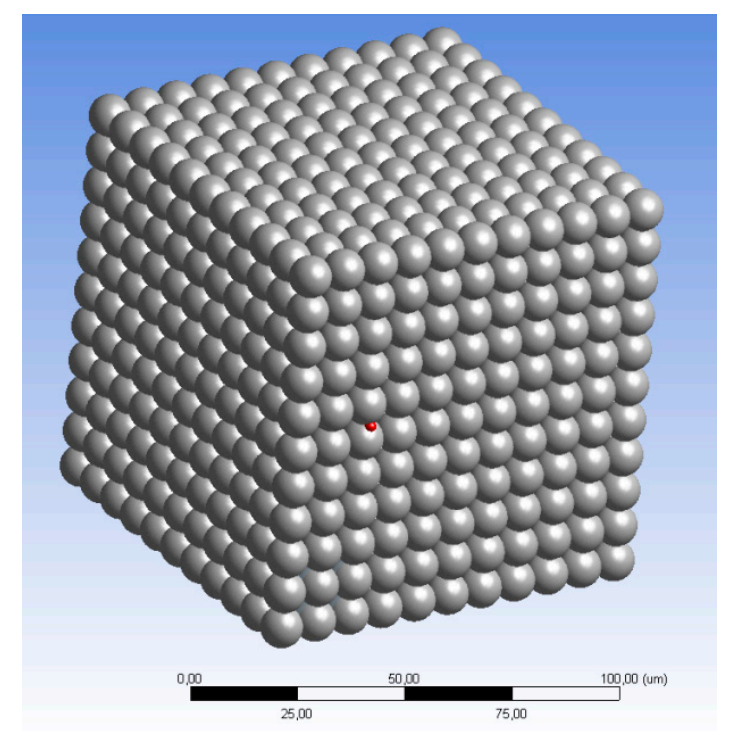

b)

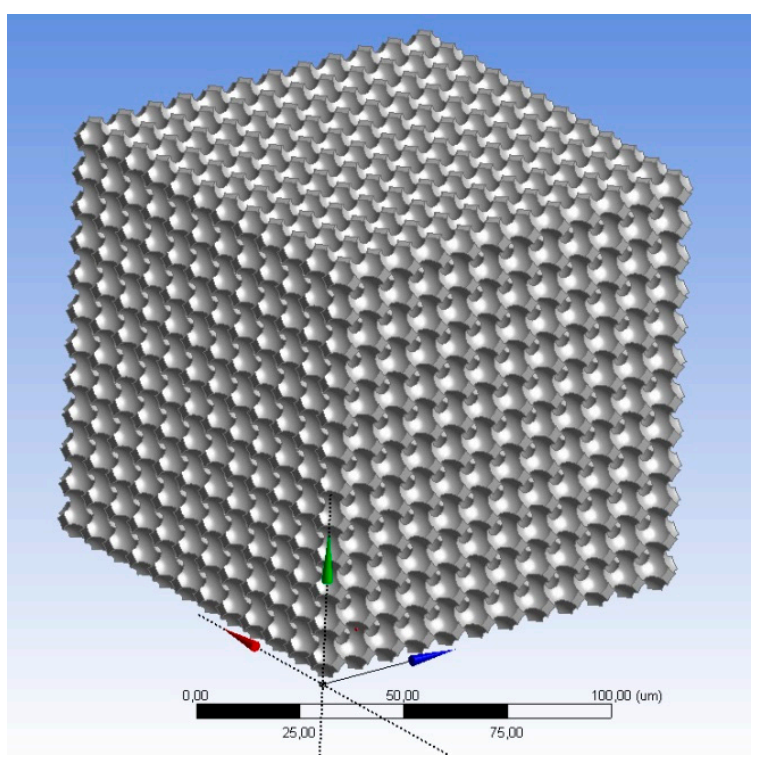

Fig. 7. Geometrical model of porous bed with a symmetric cube structure $a=20 \mathrm{~mm}, d_{\boldsymbol{k}}=10$ $\mu \mathrm{m}, \Psi=32.2 \%$ :

a) elementary bed model structure, b) geometrical structure of symmetric porous spaces. 

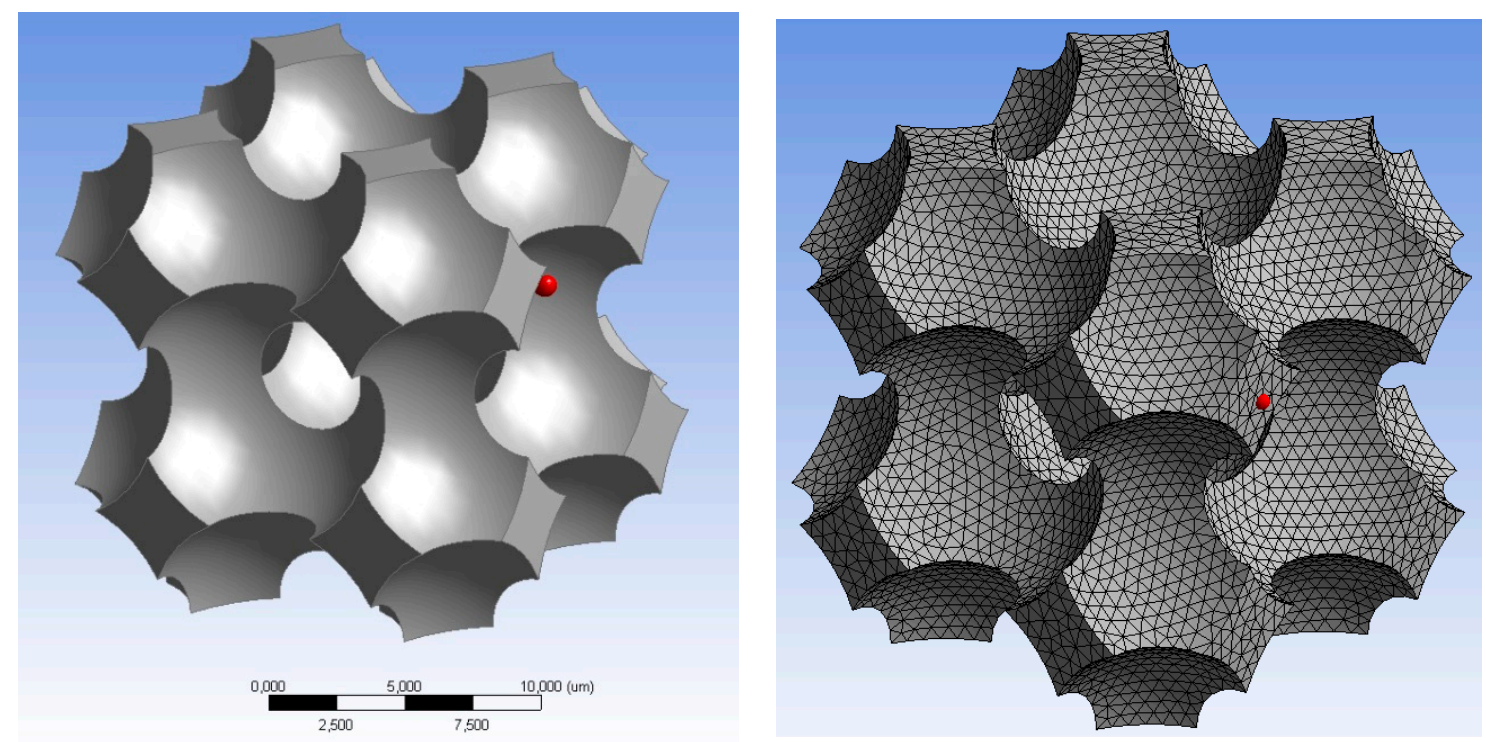

Fig. 8. Elementary unit of cellular open for flow structure:

a) model of geometry, b) calculation network $-85 \cdot 10^{3}$ cells. 


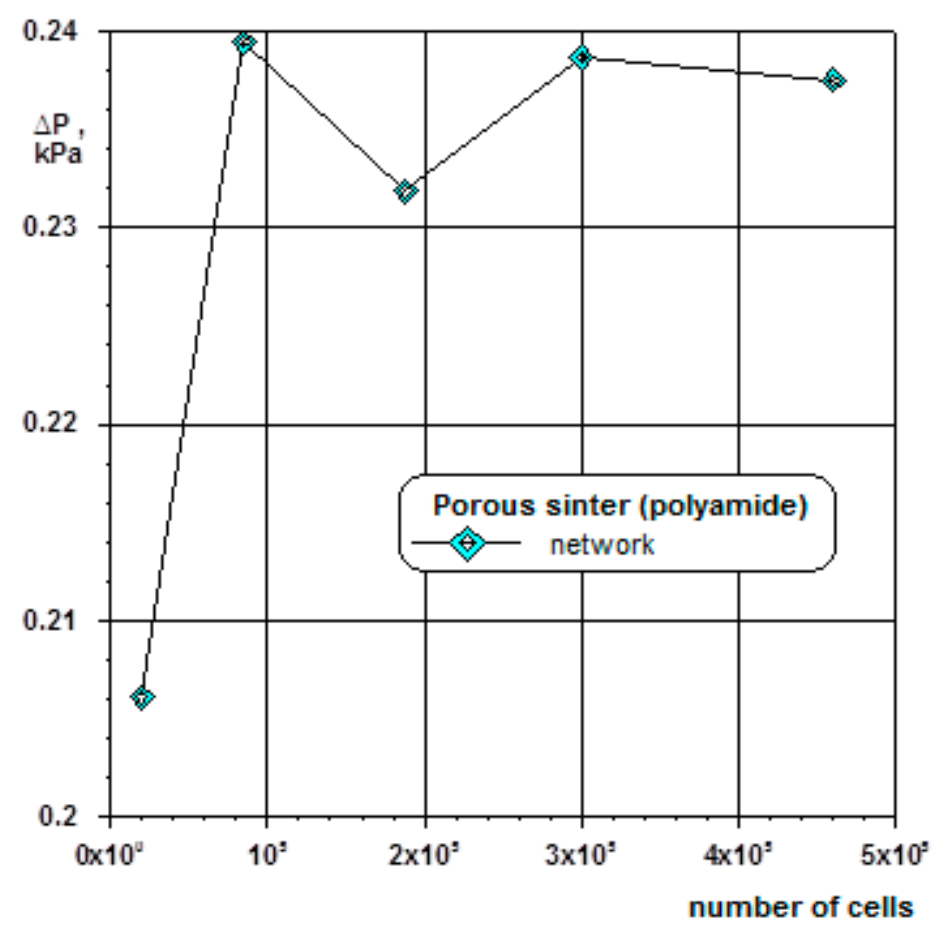

Fig. 9. Discretization of model of geometry for cube-shaped network $a=20 \mathrm{~mm}, d_{k}=10 \mu \mathrm{m}, \Psi=32.2 \%$. 
a)

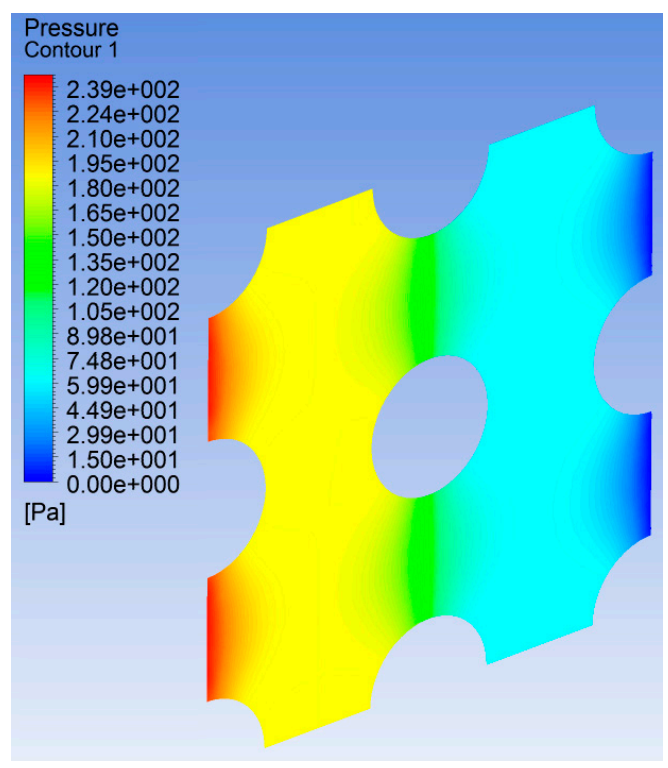

b)

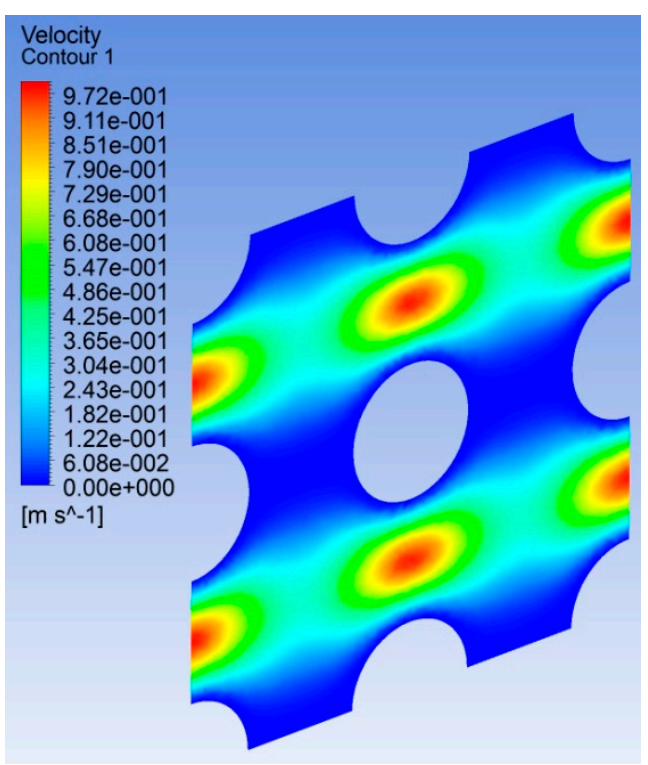

Fig. 10. Distribution of gas flow in the framework structure for the cross-section of the elementary unit: a) pressure field, b) velocity field. 
a)

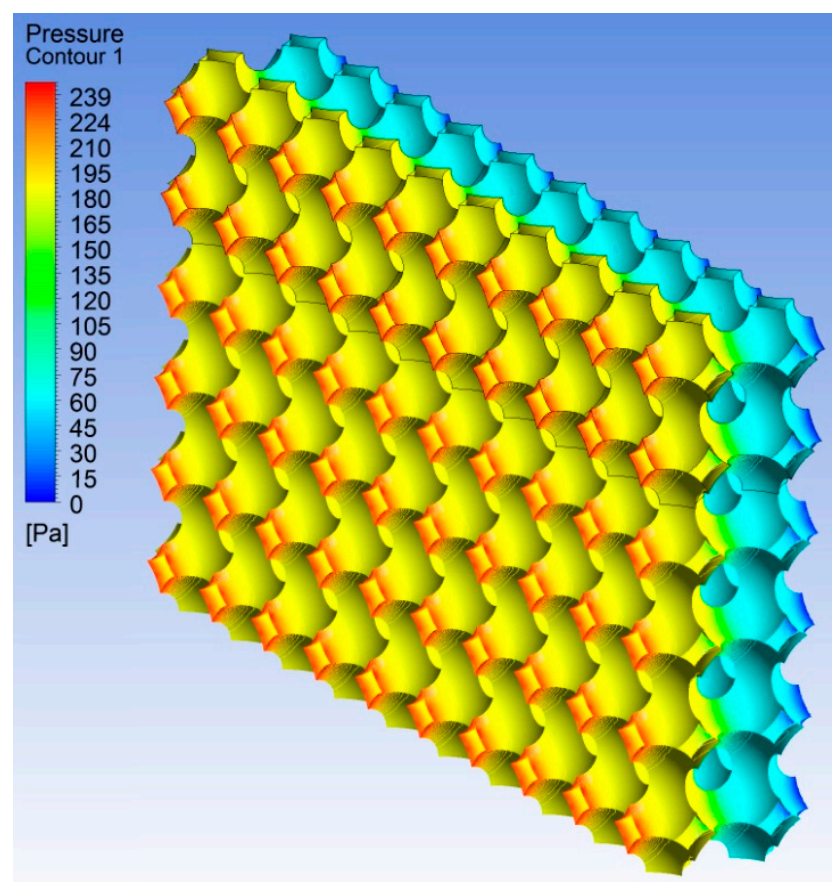

b)

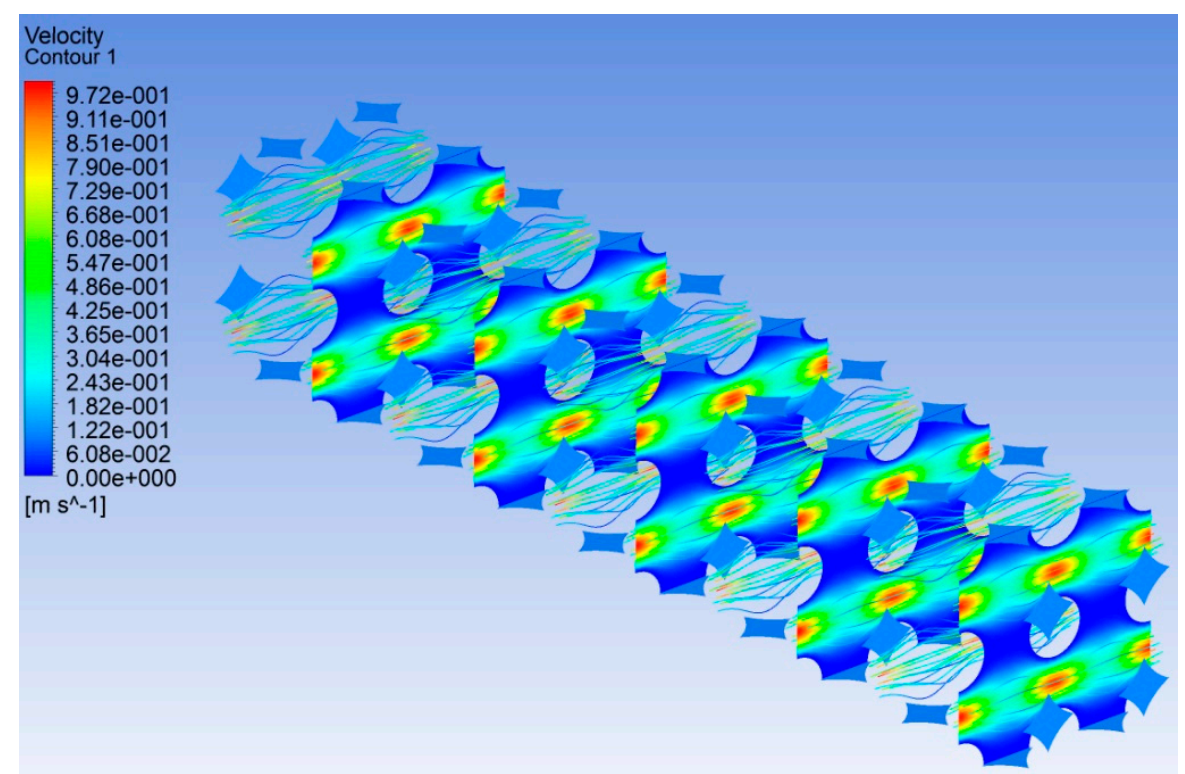

Fig. 11. Distribution of gas flow in the framework structure:

a) pressure field for the 15-times multiple of the elementary unit, b) direction lines of fluid flow and velocity profiles for 5-times multiple of the elementary unit. 


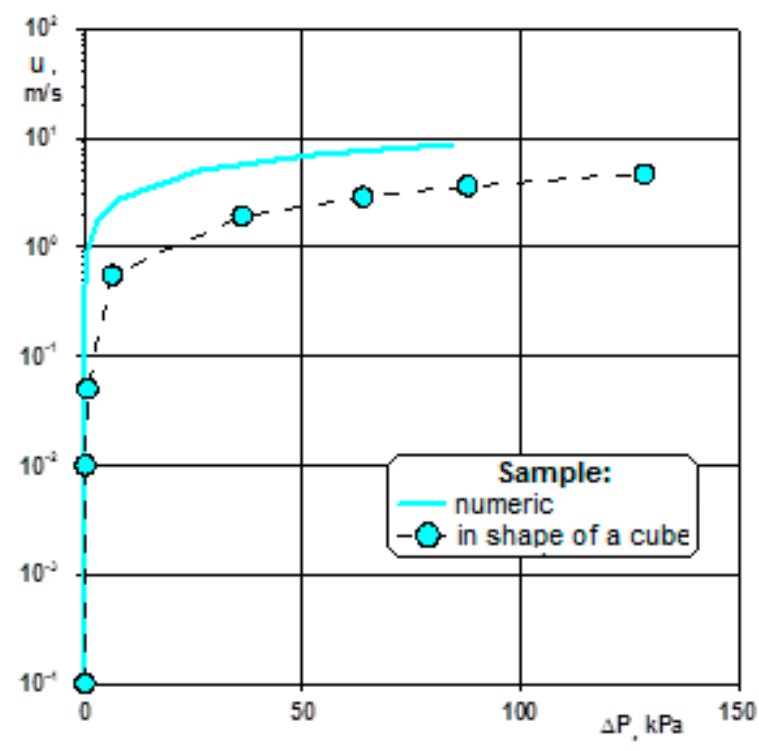

Fig. 12. Summary of experimental and numerical results for porous sinter. 


\section{Table 1}

Characteristic of research material

\begin{tabular}{|c|c|c|c|c|c|c|c|}
\hline \multirow[b]{2}{*}{$\begin{array}{l}\text { Research } \\
\text { material }\end{array}$} & \multirow[b]{2}{*}{$\begin{array}{l}\text { Porosity } \\
\text { absolute }\end{array}$} & \multirow[b]{2}{*}{$\begin{array}{l}\text { Equivalent } \\
\text { pore diameter }\end{array}$} & \multirow[b]{2}{*}{$\begin{array}{l}\text { Indicator } \\
\text { porosity }\end{array}$} & \multicolumn{2}{|c|}{ Density: } & \multicolumn{2}{|c|}{ Cubic solid: } \\
\hline & & & & apparent & skeleton & $\begin{array}{l}\text { volume } \\
\text { of the } \\
\text { sample }\end{array}$ & $\begin{array}{l}\text { equivalent } \\
\text { number of } \\
\text { volume* }\end{array}$ \\
\hline name & $\begin{array}{l}\Psi_{\mathrm{b}} \\
\%\end{array}$ & $\begin{array}{l}\mathrm{d}_{\mathrm{e}}, \\
\mu \mathrm{m}\end{array}$ & $\psi$ & $\begin{array}{l}\vartheta_{\mathrm{poz}} \\
\mathrm{kg} / \mathrm{m}^{3}\end{array}$ & $\begin{array}{l}\vartheta_{\mathrm{st}} \\
\mathrm{kg} / \mathrm{m}^{3}\end{array}$ & $\begin{array}{l}\mathrm{V} \\
\mathrm{dm}^{3}\end{array}$ & $\mathrm{n}$ \\
\hline $\begin{array}{l}\text { sintered } \\
\text { porous } \\
\text { (polyamide) }\end{array}$ & 32.3 & 10.0 & 0.5 & 772 & 1140 & 0.008 & 1 \\
\hline
\end{tabular}




\section{Table 2}

Test of research material.

\begin{tabular}{|c|c|c|c|}
\hline \multicolumn{2}{|c|}{ Research material } & \multicolumn{2}{|c|}{$\begin{array}{l}\text { Porous sinter } \\
\text { (polyamide) }\end{array}$} \\
\hline \multicolumn{2}{|c|}{ Sample number } & \multicolumn{2}{|l|}{ XV-1 } \\
\hline No. & $\begin{array}{l}\text { Reference } \\
\text { pressure } \\
\mathrm{P}_{\mathrm{o}} \\
\mathrm{MPa}\end{array}$ & $\begin{array}{l}\text { Gas } \\
\text { stream } \\
\mathrm{V} \cdot 10^{3}, \\
\mathrm{~m}^{3} / \mathrm{s}\end{array}$ & $\begin{array}{l}\text { Resistance } \\
\text { flow } \\
\Delta \mathrm{P}_{\exp }, \\
\mathrm{kPa}\end{array}$ \\
\hline \multicolumn{4}{|c|}{ direction of gas flow: $\mathrm{X}$} \\
\hline 1 & 0.04 & 0.078 & 7.2 \\
\hline 2 & 0.08 & 0.276 & 40.4 \\
\hline 3 & 0.1 & 0.376 & 63.2 \\
\hline 4 & 0.12 & 0.477 & 87.5 \\
\hline 5 & 0.16 & 0.600 & 128.6 \\
\hline \multicolumn{4}{|c|}{ direction of gas flow: Y } \\
\hline 1 & 0.04 & 0.060 & 4.7 \\
\hline 2 & 0.08 & 0.225 & 29.7 \\
\hline 3 & 0.1 & 0.380 & 66.8 \\
\hline 4 & 0.12 & 0.474 & 90.7 \\
\hline 5 & 0.16 & 0.600 & 127.7 \\
\hline \multicolumn{4}{|c|}{ direction of gas flow: $\mathrm{Z}$} \\
\hline 1 & 0.04 & 0.078 & 7.3 \\
\hline 2 & 0.08 & 0.247 & 39.1 \\
\hline 3 & 0.1 & 0.358 & 61.8 \\
\hline 4 & 0.12 & 0.452 & 85.3 \\
\hline 5 & 0.16 & 0.600 & 128.7 \\
\hline \multicolumn{4}{|c|}{ average values XYZ } \\
\hline 1 & 0.04 & 0.071 & 6.3 \\
\hline 2 & 0.08 & 0.248 & 36.1 \\
\hline 3 & 0.1 & 0.371 & 63.9 \\
\hline 4 & 0.12 & 0.467 & 87.8 \\
\hline 5 & 0.16 & 0.600 & 128.3 \\
\hline
\end{tabular}

